Editorial

\title{
Focalize the Structure of Myd88 in TLR Signaling Pathway to Modulate Innate Immune Response: New Target for Old Diseases
}

\author{
${ }^{1,2}$ Shuai Xing and ${ }^{1}$ Ping Zhou \\ ${ }^{1}$ Institute of Organ Transplantation, Tongji Hospital, \\ Tongji Medical College, Huazhong University of Science and Technology Wuhan, Hubei 430030, China \\ ${ }^{2}$ Department of Gastroenterology, Tongji Hospital, \\ Tongji Medical College, Huazhong University of Science and Technology Wuhan, Hubei 430030, China \\ Corresponding Author: Ping Zhou (pzhou@tjh.tjmu.edu.cn)
}

All diseases have their Achilles' Heel to target and this is especially true for immunorelated diseases which must be treated by targeting the immune system somewhere and somehow correctly. In the most recent decade, immunologists recognized that the Toll-Like Receptor (TLR) signaling pathway in innate immune system plays a double-edged role in the pathogenesis of many notorious diseases. Although the innate immune system functions primarily as body's first-line that ensures efficient response to foreign antigens and tolerance to self-tissues, the deterioration of the diseases above, by its nature, is an amplification and uncontrollability of the inflammatory response initiated by the innate immune system. Thus, based on innate immune system, recent studies have focused largely on the positive or negative regulatory mechanisms on how to regulate the activity of excessive inflammatory responses evolutionarily conserved family of TLRs of these inflammation-related diseases from an immunopathogenesis perspective.

The most important and the main part of immunorelated diseases is TLR hyperactivation (Akira et al., 2006). TLRs are a family of single-pass transmembrane molecules that act as key mediators for recognition of various PathogenAssociated Molecular Patterns (PAMPs) or DangerAssociated Molecular Patterns (DAMPs) and transduce the complex signaling responses for the subsequent development of adaptive immunity (Kim et al., 2008). The basic mechanism of TLR hyperactivation seems to the hyperactivation of MyD88, the main adaptor utilized by TLRs (Gay et al., 2006). The central role of MyD88 in innate immunity makes it as molecular switches in TLR activation. Although MyD88 hyperactivation is essential to counteract pathogens and prevent infections, their uncontrolled or aberrant activation can lead to pathological situations in human diseases (Lamagna et al., 2013). Thus, how to regulate MyD88 signaling by modeling the MyD88 structure is under intense investigation and these therapeutic approaches aimed at restoring proper activation will be of clinical interest.
TLRs exist as non-functional dimmers in the absence of ligand and that ligand binding induces conformational changes which allow stable receptor association (Gay et al., 2006). A conformation rearrangement of the cytoplasmic domains following ligand binding creates a scaffold to adaptor recruitment (Watters et al., 2007). The function of these adaptors is regulated by diverse mechanisms that provide the specificity to various TLR signaling pathways. Although infection, ischemia, trauma, autoimmune, allergic disorders, allo-responses and cancers involved various complex signal pathways, TLRs are involved in all of these seemingly different entities.

Further, where is the most important focal point of the MyD88 activation? Once a ligand (PAMP or DAMP) comes in contact with its TLR, the specific TLR signaling pathway is activated and performed their functions via TIR-TIR interactions. Toll/Interleukin-1 Receptor (TIR) domain is the key signaling domain to the TLR system, which is located in the cytosolic face of each TLR and their adaptor proteins. (Guven et al., 2013) The TIR-TIR crosslinkings between receptor-receptor, receptor-adaptor and adaptor-adaptor are crucial events in signal transduction. Thus it can be seen that, as the key focal point of MyD88 activation, TIR domain may be the Achilles Heel of a serious notorious diseases associated with improper response of innate immunity and so, may be a potential target for the drug discovery.

Structure determines function. There is no wonder that a major effort by the scientific community has focused on the structural network of various TIR-TIR modeling to study how function is executed and how to control. Computational structural biology of TIR domain shows it is defined by a flavodoxin-like fold containing five-stranded $\beta$ sheets ( $\beta$ A through $\beta E$ ) surrounded by five $\alpha$-helices ( $\alpha \mathrm{A}$ through $\alpha \mathrm{E}$ ) and connected together by flexible loops (AA through EE), whose portions of TIR domain could be identified structurally as an ideal target for drug discovery (Snyder et al., 2013). Currently 
available approaches for inhibition of MyD88 activity are peptides and compounds that inhibit the TIR- and DD-mediated MyD88 interactions.

Genetics and mutagenesis studies have indentified BB loop and DD loop within TIR domain as essential for each TLR signaling due to conformational flexibility. For example, the mutation to a histidine in the BB loop of TLR4 rendered $\mathrm{C} 3 \mathrm{H} / \mathrm{HeJ}$ mice non-responsive to LPS; (Piao et al., 2013) the DD-loop of TLR2 and the BB-loop of TLR1 have been shown to participate in TLR1/TLR2 heterodimerization (Gautam et al., 2006b); similarly, the TIR interactions between TLR2 and MyD88 are required for the two proteins to interact (Sahoo et al., 2013); peptides (TcpC) targeting on the BB loop of MyD88 and other adaptors have been indentified that modulate host immune responses through their TIR domains (Snyder et al., 2013). Furthermore, modeling and functional studies revealed that some other regions outside of the two loops above also play vital roles in TIR domain associations or TLR signaling, such as CD, DD, EE loops and $\alpha$ E-helix (Snyder et al., 2013). A putative model might therefore be that TLR dimerization is dependent on the space conformation and electronic cloud distribution of TIR domain and that the conformational changes in the key portion of TIR domain directly influences the electrostatic surfaces that is required for TIR-TIR interactions. Ultimately, the hope is that inhibitors can be designed to interfere specifically with the conformation and alter the electrostatic complementarity of TIR domain, which may be an attractive therapeutic approach for the treatment of different human diseases correlated with MyD88 hyperactivation. As identified by our published data, we have generated a series of small compound inhibitors of MyD88 designed after the structure of MyD88 TIR domain, which combine to the residues in DD loop, $\mathrm{aE}$ and the Poc site I179 of BB loop, inhibits the homodimerization of MyD88. The inhibitor showed astonishing excellent effects in preventing and/or treating transplant rejection (Li et al., 2017), Graft Versus Host Disease (GVHD), ischemia and reperfusion injury (Zhang et al., 2016a) type I diabetes (Zhang et al., 2016b) and inflammation associated cancer (Xie et al., 2016).

Although function of TIR-TIR interactions to initiate and propagate inflammation makes TIR domain attractive therapeutic target, there is still a fierce debate on whether TIR abrogation could inhibit the immune responses overly and thoroughly, resulting in the development of an overwhelming infection and defective immunological surveillance. Just as the dual function of MyD88 signaling in intestine, MyD88 signaling in IECs promotes expression of selective antimicrobial peptide, which associate enhanced host resistance and survival, but after infection with the $\mathrm{H}$ hepaticus, innate cell-derived MyD88 signaling promote inflammatory gene expression leading to colitis and extra-intestinal inflammation. In nature, the binding surfaces or interfaces of TIR domain are highly conserved (Gerdol et al., 2017). That is, different proteins containing TIR domain in TLR signaling world use the similar interfaces to interact with their partners. Thus, previously identified interfaces and occupied the binding sites could be used to block all TIR-TIR interactions. However, accumulating evidence has shown that lifethreatening infection or cancer occurrence was rarely seen in experimental animals that bearing TIR blockage in previous studies (Loiarro et al., 2007; 2013) and in our own experience (Fig. 1). The possible explanation of the phenomenon is that the sensing, signaling and responding of innate immunity were not absolutely abrogated, or that some compensated signaling pathway exists when TLR pathway is inhibited. Thanks to the limitation and selectivity of TLR inhibition and compensatory response of natural immunity. To achieve the therapeutic goal preferably and avoid possible side effect, more comprehensive and precise mechanisms underlying the TIR-abrogating double-edge effects in different animal models should be elucidated.

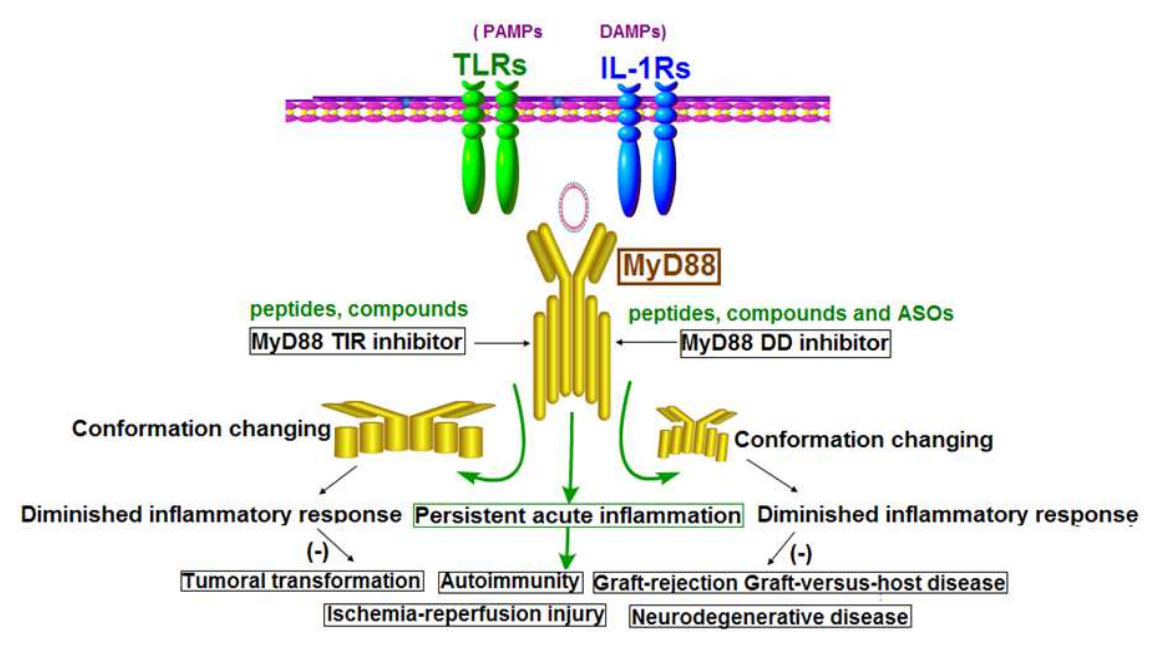

Fig. 1. Focalize TIR domain to modulate innate immune responses: New target for old diseases 


\section{Author's Contributions}

Shuai Xing: Contributed in drafting the article and reviewing it critically for significant intellectual content.

Ping Zhou: Designed the research plan, organized the study and given final approval of the version to be submitted.

\section{Conflict of Interest}

Authors declare no conflict of interest.

\section{References}

Akira, S., S. Uematsu and O. Takeuchi, 2006. Pathogen recognition and innate immunity. Cell, 124: 783-801. DOI: 10.1016/j.cell.2006.02.015

Gautam, J.K., Ashish, L.D. Comeau, J.K. Krueger and M.J. Smith, 2006b. Structural and functional evidence for the role of the TLR2 DD loop in TLR1/TLR2 heterodimerization and signaling. J. Biol. Chem., 281: 30132-30142. DOI: $10.1074 / \mathrm{jbc} . M 602057200$

Gay, N.J., M. Gangloff and A.N. Weber, 2006. Toll-like receptors as molecular switches. Nat. Rev. Immunol., 6: 693-698. DOI: 10.1038/nri1916

Gerdol, M., P. Venier, P. Edomi and A. Pallavicini, 2017. Diversity and evolution of TIR-domain-containing proteins in bivalves and Metazoa: New insights from comparative genomics. Dev. Comp. Immunol., 70: 145-164. DOI: 10.1016/j.dci.2017.01.014

Guven, M.E., O. Keskin, A. Gursoy and R. Nussinov, 2013. The structural network of inflammation and cancer: merits and challenges. Semin. Cancer Biol., 23: 243-251.

DOI: 10.1016/j.semcancer.2013.05.003

Kim, I.K., D.S. Bedi, C. Denecke, X. Ge and S.G. Tullius, 2008. Impact of innate and adaptive immunity on rejection and tolerance. Transplantation, 86: 889-894. DOI: 10.1097/TP.0b013e318186ac4a

Lamagna, C., P. Scapini, J.A. van Ziffle, A.L. DeFranco and C.A. Lowell, 2013. Hyperactivated MyD88 signaling in dendritic cells, through specific deletion of Lyn kinase, causes severe autoimmunity and inflammation. Proc. Natl. Acad. Sci. USA., 110: E3311-E3320. DOI: 10.1073/pnas.1300617110

Li, C., L.M. Zhang, X. Zhang, X. Huang and Y. Liu et al., 2017. Short-term pharmacological inhibition of MyD88 homodimerization by a novel inhibitor promotes robust allograft tolerance in mouse cardiac and skin transplantation. Transplantation, 101: 284-293.

DOI: $10.1097 /$ TP.0000000000001471
Loiarro, M., F. Capolunghi, N. Fanto, G. Gallo and S. Campo et al., 2007. Pivotal advance: Inhibition of MyD88 dimerization and recruitment of IRAK1 and IRAK4 by a novel peptidomimetic compound. J. Leukoc. Biol., 82: 801-810. DOI: $10.1189 /$ jlb. 1206746

Loiarro, M., V. Ruggiero and C. Sette, 2013. Targeting the toll-like receptor/interleukin 1 receptor pathway in human diseases: Rational design of MyD88 inhibitors. Clin. Lymphoma Myeloma Leuk., 13: 222-226. DOI: 10.1016/j.clml.2013.02.003

Piao, W., S.N. Vogel and V.Y. Toshchakov, 2013. Inhibition of TLR4 signaling by TRAM-derived decoy peptides in vitro and in vivo. J. Immunol., 190: 2263-2272. DOI: 10.4049/jimmunol.1202703

Sahoo, B.R., M. Basu, B. Swain, M.R. Dikhit and P. Jayasankar et al., 2013. Elucidation of novel structural scaffold in rohu TLR2 and its binding site analysis with peptidoglycan, lipoteichoic acid and zymosan ligands and downstream MyD88 adaptor protein. BioMed Res. Int., 2013: 185282-185296. DOI: $10.1155 / 2013 / 185282$

Snyder, G.A., C. Cirl, J. Jiang, K. Chen and A. Waldhuber et al., 2013. Molecular mechanisms for the subversion of MyD88 signaling by TcpC from virulent uropathogenic Escherichia coli. Proc. Natl. Acad. Sci. USA., 110: 6985-6990. DOI: $10.1073 /$ pnas. 1215770110

Watters, T.M., E.F. Kenny and L.A. O'Neill, 2007. Structure, function and regulation of the Toll/IL-1 receptor adaptor proteins. Immunol. Cell Biol., 85: 411-419. DOI: 10.1038/sj.icb.7100095

Xie, L., F.C. Jiang, L.M. Zhang, W.T. He and J.H. Liu et al., 2016. Targeting of MyD88 homodimerization by novel synthetic inhibitor TJ-M2010-5 in preventing colitis-associated colorectal cancer. J. Natl. Cancer Inst., 108: djv364-djv364. DOI: 10.1093/jnci/djv364

Zhang, L.M., J.H. Liu, C.B. Xue, M.Q. Li and S. Xing et al., 2016. Pharmacological inhibition of MyD88 homodimerization counteracts renal ischemia reperfusion-induced progressive renal injury in vivo and in vitro. Sci. Rep., 6: 26954-26954. DOI: $10.1038 /$ srep26954

Zhang, X., S. Xing, M. Li, L. Zhang and L. Xie et al., 2016. Beyond knockout: A novel homodimerizationtargeting MyD88 inhibitor prevents and cures type 1 diabetes in NOD mice. Metabolism, 65: 1267-1277. DOI: 10.1016/j.metabol.2016.05.005 